ORIGINAL ARTICLE

\title{
Effect of gluten-free diet and adherence on growth and diabetic control in diabetics with coeliac disease
}

\author{
O I Saadah, M Zacharin, A O'Callaghan, M R Oliver, A G Catto-Smith
}

Arch Dis Child 2004;89:871-876. doi: 10.1136/adc.2002.012799

\begin{abstract}
See end of article for authors' affiliations

....................

Correspondence to: Associate Professor A Catto-Smith, Department of Gastroenterology and Clinical Nutrition, Royal Children's Hospital, Flemington Road, Parkville, Victoria 3052, Australia; tony. cattosmith@rch.org.au
\end{abstract}

Accepted 28 January 2004

\begin{abstract}
Aims: To study the effect of gluten-free diet on growth and diabetic control of children with type 1 diabetes mellitus and coeliac disease.

Methods: Twenty one children (mean age 7.5 years, range 1.6-12.9) with type 1 diabetes, primarily initially identified on the basis of symptoms and consecutively diagnosed with coeliac disease by biopsy over a 10 year period, were matched by sex, age at onset, and duration of diabetes with two diabetic controls without coeliac disease. Weight, height, haemoglobin Alc, and insulin requirements were measured before and for 12 months after the diagnosis and treatment of coeliac disease. Dietary awareness and adherence were assessed by structured questionnaire.

Results: A gluten-free diet resulted in a significant increase in weight-for-age z scores at 12 months after diagnosis (mean increase in z score 0.33) and in BMI (mean increase in z score 0.32). Increases in height did not achieve statistical significance. Controls showed no significant changes in weight, height, or BMI over the same period. Insulin dosage at diagnosis was less in coeliacs than in controls (mean difference 0.16 units $/ \mathrm{kg} /$ day), but was similar to controls once a gluten-free diet had been established. Questionnaires were obtained in 20 patients. There appeared to be a relation between dietary awareness/adherence and growth parameters, but the small number of patients with "poor/fair" dietary adherence prevented meaningful analysis of this group.

Conclusion: Identification and dietary treatment of coeliac disease in children with diabetes improved growth and influenced diabetic control. Evaluation of the outcome of treatment of coeliac disease in diabetics should include assessments of gluten intake.
\end{abstract}

$\mathrm{T}$ he association between coeliac disease (CD) and type 1 diabetes mellitus was identified in 1969. ' Subsequently, the prevalence of $\mathrm{CD}$ in children with type 1 diabetes mellitus has been established to be about $2-5 \%{ }^{2-7}$ A relation between the two conditions can in part be explained on the basis of a similar genetic background. Class II HLA DR3 and HLA DQ2 genotypes are frequently seen in both disorders. ${ }^{8}{ }^{9}$

Many diabetic clinics now either maintain a high clinical suspicion or routinely screen patients for CD using serological markers. Following a finding of two or more positive serological markers and typical changes on a duodenal biopsy, CD may then be diagnosed in the absence of significant clinical symptoms. However, the practical limitations imposed by gluten restriction, in addition to that of a diabetic diet, place considerable restrictions on the lifestyle of a child. Furthermore, there is very little data available on the clinical outcome of treatment of CD in this situation, where symptoms are often minimal or absent. While some already published studies show a benefit to gluten restriction on growth and parameters of diabetic control, ${ }^{10}$ others show either questionable or no clinical improvement. ${ }^{11-13}$ All are based on small numbers and none have evaluated outcome in relation to dietary adherence.

The purpose of our study was to evaluate the effect of dietary awareness and adherence to a gluten-free diet (GFD) on growth and diabetic control in diabetics with newly diagnosed $\mathrm{CD}$. The diagnosis of coeliac disease in patients identified for this study predated the introduction of routine serological screening for coeliac disease in our diabetic clinic.

\section{PATIENTS AND METHODS}

We identified all patients with type 1 diabetes who had been diagnosed with $\mathrm{CD}$ at the Royal Children's Hospital, Melbourne between September 1989 and December 1999.
Data were obtained by reviewing the medical record of each patient. These patients were identified as having CD before routine serological screening was carried out in our diabetic population as part of their outpatient management. Most patients included in this study had either gastroenterological symptoms or evidence of blood sugar levels which were difficult to control with standard doses of insulin before the diagnosis of $\mathrm{CD}$.

Small bowel biopsy specimens were obtained by upper gastrointestinal endoscopy with 2-4 specimens from the distal duodenum, and interpreted by one pathologist. Coeliac disease was diagnosed in all on the basis of duodenal histology. At the time of diagnosis, all patients received education about a gluten-free diet from an experienced paediatric dietician.

Patients attended their diabetic clinic every three months for follow up. Serial measurements of weight, height, haemoglobin Alc level, and insulin requirement were obtained from the medical record for a one year period after starting on a gluten-free diet. These were compared to the measurements obtained immediately before the diagnosis of CD. The z scores for weight-for-age, height-for-age, and body mass index (BMI) were calculated by using an anthropometric software program (EpiInfo 2002, CDC 2000, Centers for Disease Control and Prevention, Atlanta, GA, USA). Growth and diabetic control data were recorded at 3, 6, 9, and 12 months after starting the gluten-free diet.

Dietary awareness and adherence to the gluten-free diet were assessed through a structured telephone questionnaire, which was conducted by a dietician with experience in educating children and families of children with CD. This assessment was completed at a single time point during the

Abbreviations: $B M I$, body mass index; $C D$, coeliac disease; GFD, gluten-free diet; $\mathrm{HbAlc}$, haemoglobin $\mathrm{Alc}$ 
12 month study period (1999-2000) while on a gluten-free diet. The questionnaire was designed to assess the parent's and/or child's knowledge of the dietary requirements of CD, sources of gluten, frequency and amount of gluten consumption, contacts with a dietician and a Coeliac Society, and attitude and motivation towards a gluten-free diet. Each questionnaire was scored, and then classified into one of four categories:

- Excellent (A) for those who were very highly aware of the requirements of a gluten-free diet and very adherent all the time

- Good (B) where they were aware of dietary needs and adherent most of the time, with occasional intake of small amounts of gluten

- Fair (C) where their awareness of the requirements of a gluten-free diet was poor and they were often nonadherent, regularly consuming small amounts of gluten

- Poor (D) where they had little understanding of the requirements of a gluten-free diet, and were very nonadherent, often consuming significant amounts of gluten (full questionnaire available from authors).

Each child with coeliac disease was matched for age, sex, and duration of diabetes with two diabetic control subjects who did not have evidence of coeliac disease.

Statistical analysis was performed using Graph Pad InStat (V3.05 for Windows 95, GraphPad Software, San Diego, California, USA). Comparisons between groups at specific time points were made using $t$ tests. Multiple comparisons within each patient group after commencing a gluten-free diet were made by repeated measures ANOVA with post hoc analysis using Dunnett's test. Paired $t$ tests were used for comparisons between baseline and 12 months. Results are expressed as mean (SD) unless stated otherwise. Statistical significance was accepted if the two tailed $p$ value was less than 0.05 .

\section{RESULTS}

Twenty six patients with type 1 diabetes and CD were identified. We excluded five children from further analysis: one female in whom CD had been diagnosed five years before the development of type 1 diabetes; three (two males) with Down's syndrome; and one female who had been lost to follow up and in whom no clinical data were available on growth or diabetic control. We report on the remaining 21 children. Eight were male (M:F 0.62:1.0). Their mean age at diagnosis of type 1 diabetes was 4.0 (2.7) years (range 0.9-
9.9), and at diagnosis of CD was 7.5 (3.0) years (range 1.612.9). The median duration of type 1 diabetes before diagnosis of CD was 2.9 years (range 0.2-10.8). Of the 21 children, 20 (95\%) had reported at least some symptoms suggestive of possible gastrointestinal pathology prior to the diagnosis of $\mathrm{CD}$ : diarrhoea $(\mathrm{n}=11)$, abdominal pain $(n=11)$, abdominal distension $(n=6)$, and poor weight gain $(\mathrm{n}=7)$. Definite symptomatic improvement was recorded in 10 of the $21(48 \%)$ after starting the gluten-free diet. One other child had had a marked increase in insulin requirements.

Although serological testing had been obtained in all 21 patients, the inclusion period of the study partially predated the availability of all current serological tests. Antigliadin antibodies had been obtained in all 21 patients and antiendomysial antibodies in 16. Antiendomysial antibodies were negative in two patients, but both had raised antigliadin IgA and IgG antibodies. Coeliac disease was ultimately diagnosed on the basis of characteristically abnormal duodenal histology in all apart from one patient. This child had a biopsy that was reported as showing "duodenitis", but also had abnormal antigliadin serology. A gluten-free diet was commenced and there was an improvement in growth parameters. The histology of a duodenal biopsy obtained seven months later was completely normal. This child subsequently continued a gluten-free diet.

We compared clinical and anthropometric parameters before and after commencement of a gluten-free diet. The mean weight-for-age $\mathrm{z}$ score at diagnosis of CD was 0.43 (1.27) $(95 \%$ CI $-0.15,1.01)$. The mean weight-for-age z score at 12 months $(0.76,95 \%$ CI $0.25,1.27)$ had increased significantly $(\mathrm{p}=0.049$ ) (fig $\mathrm{l}$ ). The mean height-for-age $\mathrm{z}$ score at diagnosis of CD was 0.11 (1.06) (95\% CI -0.37 , 0.59). After 12 months, the mean height increased, although this was not convincingly consistent (height-for-age z score at 12 months, $0.27(0.95), 95 \% \mathrm{CI}-0.18,0.74 ; \mathrm{p}=0.14)$. BMI had increased significantly by 12 months after the diagnosis of CD (BMI-for-age z score at diagnosis was 0.44 (1.16), 95\% CI $-0.10,0.98$; compared to score at 12 months of 0.76 (0.89), 95\% CI 0.36, 1.16; p =0.015). Matched controls were heavier and taller than children with coeliac disease at diagnosis, although this was not convincingly consistent (mean difference in weight $\mathrm{z}$ score $-0.58,95 \% \mathrm{CI}-1.05,0.13$, $\mathrm{p}=0.12$; mean difference in height $\mathrm{z}$ score $-0.25,95 \% \mathrm{CI}$ $-0.82,0.31, \mathrm{p}=0.35$; mean difference in BMI-for-age $\mathrm{z}$ score $-0.42,95 \%$ CI $-0.99,0.15, \mathrm{p}=0.14)$. Control groups showed no evidence of significant change in weight, height or BMI over the study period.
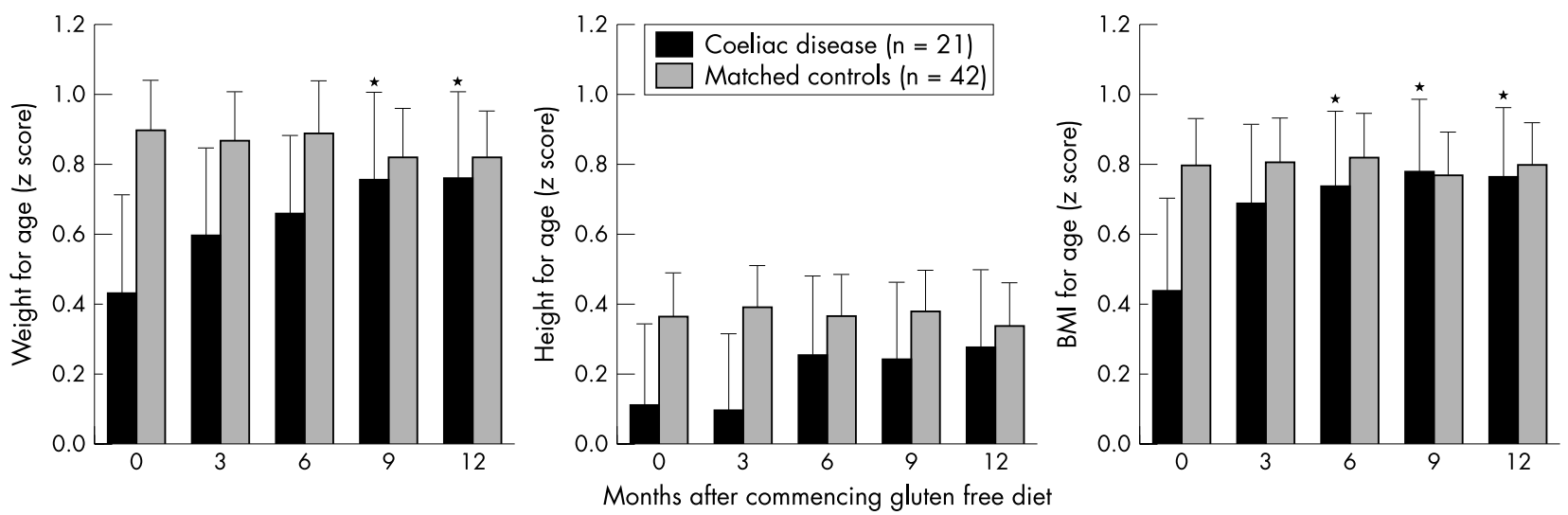

Figure 1 Weight, height, and BMI standard deviation scores for age after diagnosis of coeliac disease in type 1 diabetics and in matched diabetic controls without coeliac disease. There were statistically significant increases in weight-for-age at 9 and 12 months and in BMl at 6,9, and 12 months. ${ }^{*} p<0.05$ compared to parameter at diagnosis. 


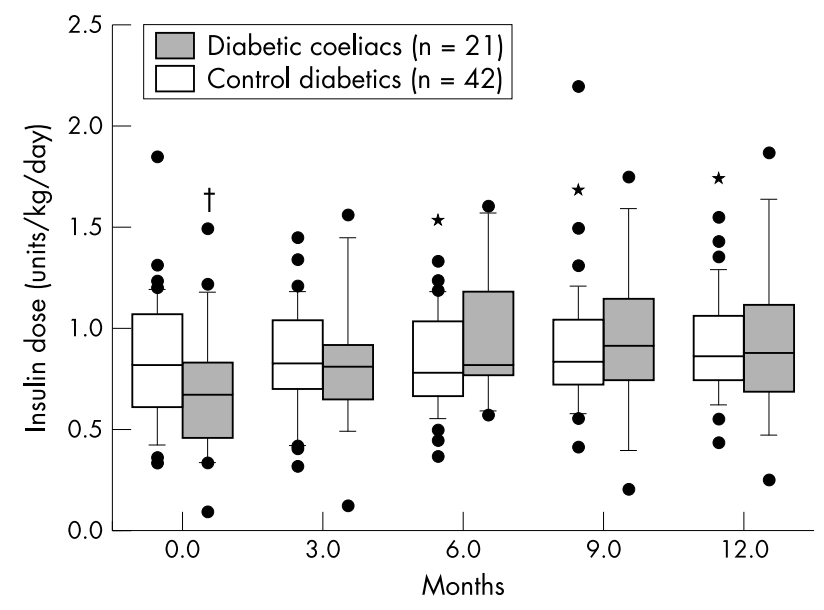

Figure 2 Insulin dose after diagnosis of coeliac disease compared to controls. Insulin dosage increased significantly after diagnosis of coeliac disease. ${ }^{*} p<0.05$ compared to insulin dose at diagnosis; $\uparrow p=0.05$ compared to control.

There was a significant increase in the mean daily dose of insulin between diagnosis of CD $(0.67(0.32)$ units $/ \mathrm{kg} / \mathrm{day})$ and that at 6, 9, and 12 months (0.96 (0.34), 0.95 (0.37), 0.94 (0.38), respectively) (fig 2). At diagnosis of CD, insulin dosage was less than in controls $(0.84(0.25)$ units $/ \mathrm{kg} /$ day; mean difference in insulin dose $-0.24,95 \%$ CI $-0.48,0.0051$, $\mathrm{p}=0.054)$. Insulin dose was similar to controls once a glutenfree diet had been established. Haemoglobin Alc levels did not differ significantly with commencement of a gluten-free diet (at diagnosis $8.03(0.86) \%$; 3 months $8.00(0.10) \%, 6$ months 8.17 (1.18)\%, 9 months $7.68(0.58) \%, 12$ months 8.04 $(0.98) \%)$, and were similar to controls at matched periods (8.21 (1.19)\%; 8.28 (1.34)\%; 8.49 (0.97)\%; 8.12 (1.32)\%; 8.08 $(0.74) \%)$.

We examined whether there were significant differences between the groups that did or did not show symptomatic improvement. There were no significant differences between these two groups in anthropometric measures or insulin dosage before or after diagnosis of coeliac disease. Despite the apparent absence of recorded symptomatic improvement, children in this group still had a significant increase in weight after commencing a gluten-free diet $(p=0.009)$.
Questionnaires were obtained from 20 patients at a median interval of 19 months (range 3-112 months) after diagnosis of coeliac disease. Of these 20 patients, dietary adherence was graded as " $\mathrm{A}$ " in 5 , " $\mathrm{B}$ " in 12 , " $\mathrm{C}$ " in 1 , and " $\mathrm{D}$ " in 2 . Symptoms resolved in 2 of 5 patients in group A, 6 of 12 in group B, 1 of 1 in group C, and 1 of 2 in group D. Because of the small numbers in groups $\mathrm{C}$ and $\mathrm{D}$, these were combined for further analysis. There were no significant differences between these groups in age at diagnosis or duration for either diabetes or of coeliac disease. We examined the impact of dietary adherence in growth parameters and diabetic control.

\section{Growth}

Group A

The weight-for-age (fig 3) of children who had "excellent" adherence (group A) was not significantly less than matched controls at diagnosis $(p=0.59)$, but did increase over the 12 months following diagnosis (mean difference $0.58,95 \% \mathrm{CI}$ $1.50,-0.33 ; \mathrm{p}=0.15)$. This linear trend was statistically significant $(p=0.011)$. The height-for-age of children from group A was not significantly different from that of matched controls. Height-for-age (fig 4) showed no evidence of a significant change with time after diagnosis of coeliac disease. The BMI-for-age of children who had "excellent" adherence (group A) increased slightly over the 12 months following diagnosis (mean difference 0.51 , 95\% CI 1.34, $-0.31 ; p=0.16)$. Children who subsequently had excellent adherence (group A) had a significantly lower BMI-for-age (fig 5) at diagnosis compared to their matched controls $(\mathrm{p}=0.047)$. There was no significant difference by 12 months after diagnosis compared to matched controls. Children with "excellent" adherence also appeared to have a lower BMIfor-age at diagnosis than the other adherence groups, but this was not convincingly consistent (ANOVA, $\mathrm{p}=0.18$ ).

\section{Group B}

Children with "good" adherence had a lower weight, height, and BMI-for-age at diagnosis than matched controls (figs 35), although this failed to reach statistical significance (weight $p=0.073$, height $p=0.11$, BMI $p=0.13$ ). Twelve months later, there were increases in weight-for-age $(0.19$, $95 \%$ CI $-0.15,0.52 ; p=0.24)$, height-for-age $(0.07,95 \%$ CI $-0.14,0.28 ; \mathrm{p}=0.14)$, and BMI-for-age $(0.30,95 \% \mathrm{CI}-0.17$, $0.78 ; \mathrm{p}=0.19)$, although these were not convincingly consistent.
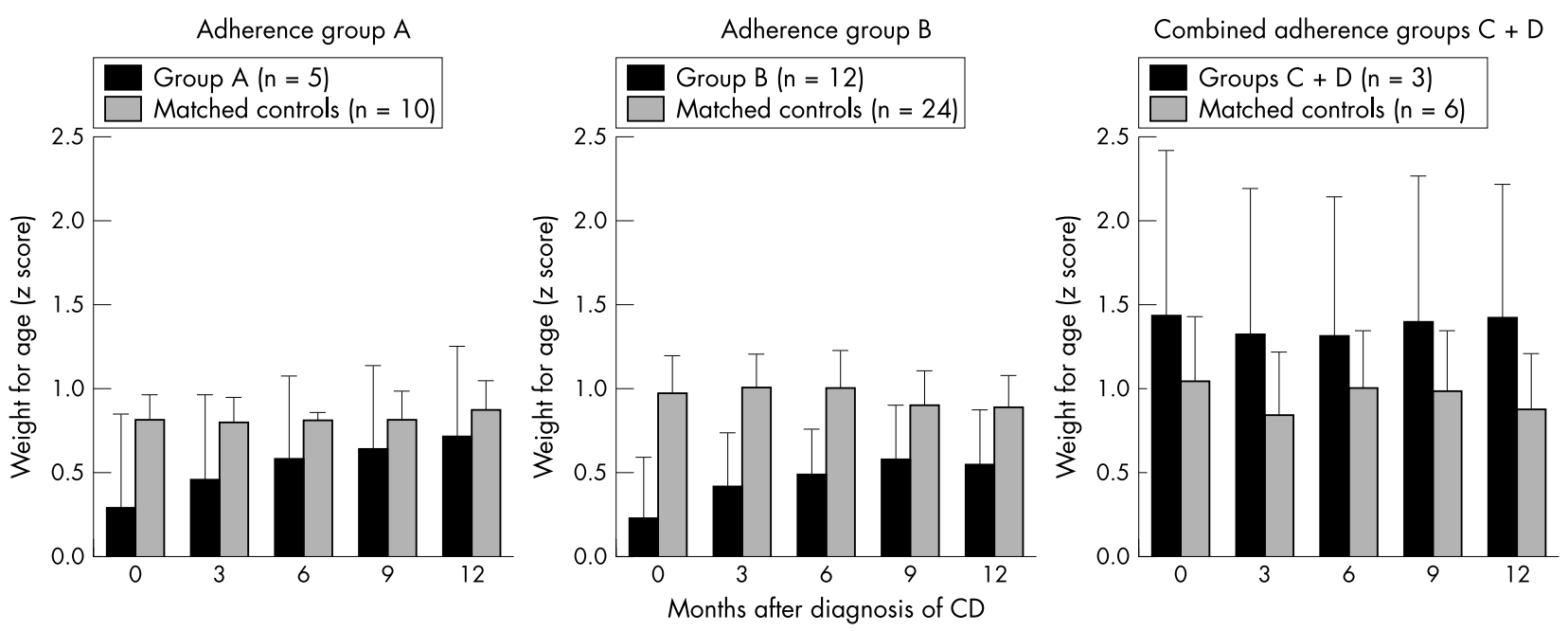

Figure 3 Weight-for-age after diagnosis of coeliac disease by dietary adherence. " $A$ " represented excellent adherence, " $B$ " good adherence, and "CD" was assessed as poor to fair. 

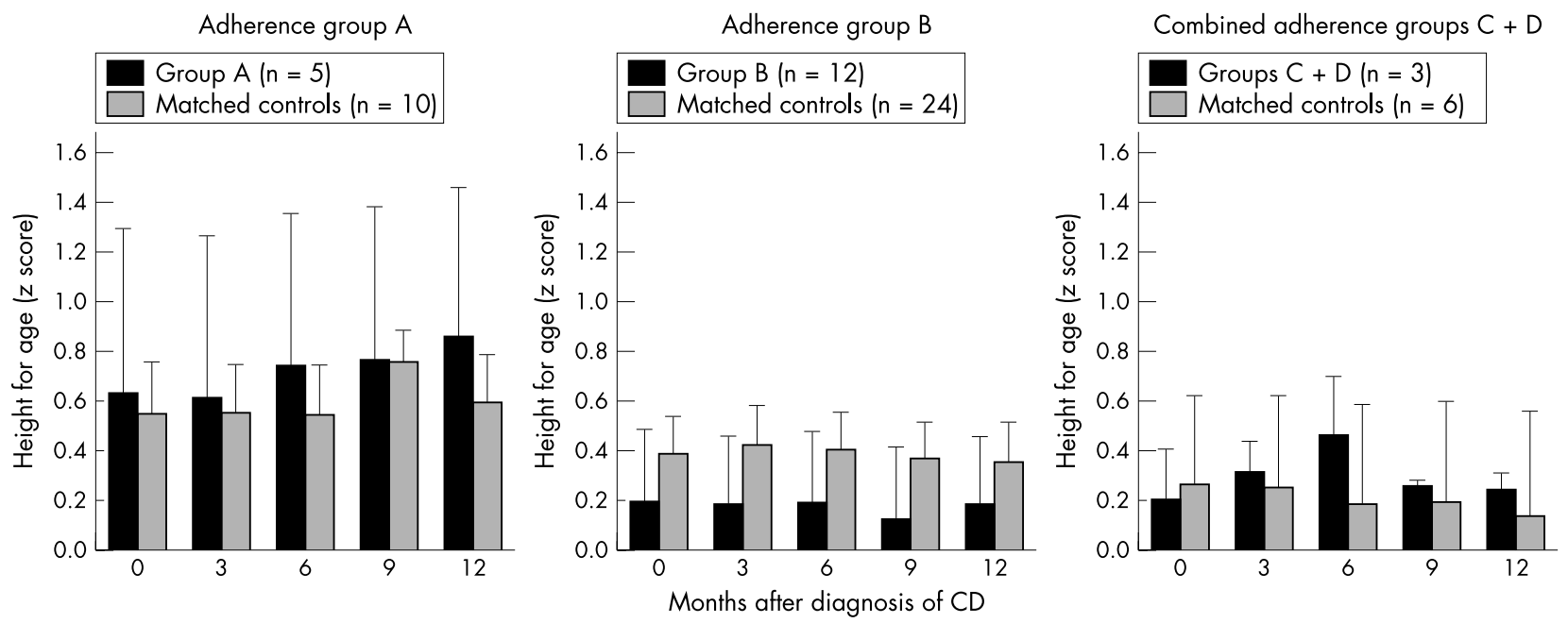

Figure 4 Height-for-age after diagnosis of coeliac disease by dietary adherence. " $\mathrm{A}$ " represented excellent adherence, " $\mathrm{B}$ " good adherence, and "CD" was assessed as poor to fair.

\section{Groups C and D}

There were only three children with "fair" or "poor" adherence. Their weight, height, and BMI-for-age were not significantly different to matched controls at diagnosis (weight $p=0.66$, height $p=0.92$, BMI $p=0.71$ ). There was no evidence of any significant change in these parameters with time after diagnosis of coeliac disease.

\section{DISCUSSION}

Suboptimal therapeutic control of both CD and type I diabetes is known to lead to impairment in growth and substantial morbidity. Effective treatment of each is only achieved with lifelong restrictive diets. Those patients who have both $C D$ and type 1 diabetes are faced with a doubly difficult regimen. Non-adherence to the gluten-free diet among patients with CD and type 1 diabetes is reported to be common, with one study finding only $30 \%$ who complied with a strict gluten-free diet. ${ }^{1-18}$ At least one study ${ }^{13}$ also found no evidence that dietary adherence improved growth in diabetics with $\mathrm{CD}$.

Routine serological surveys of patients with type 1 diabetes have identified patients with abnormal serology and duodenal histology, ${ }^{12}$ but often with minimal gastrointestinal symptoms. One small study ${ }^{10}$ found improvement in BMI and HbAlC, but others have found little apparent benefit to the patient in diagnosing asymptomatic coeliac disease. ${ }^{11}$ If this is accepted, it is difficult for such patients to accept the necessity for a life long gluten-free diet in addition to the demands placed on them by treatment of their diabetes.

Most of our patients were reported to have some symptoms that might be related to a gastrointestinal pathology, but these symptoms only improved in about 50\%, despite good dietary adherence. Referral for assessment of possible coeliac disease was initiated by the diabetologist at a low threshold of suspicion. It is likely that some of these symptoms were not related to coeliac disease. Importantly, however, those who failed to show symptomatic improvement still showed a significant increase in weight. This emphasises the relatively "silent" impact of coeliac disease.

Dietary adherence was good in about $80 \%$ of our patients. This is better than that reported in other studies of children with type 1 diabetes who have coeliac disease, ${ }^{13}$ and comparable to the best rates observed in non-diabetic coeliacs (56-81\% good adherence). ${ }^{14-18}$ Somewhat surprisingly,
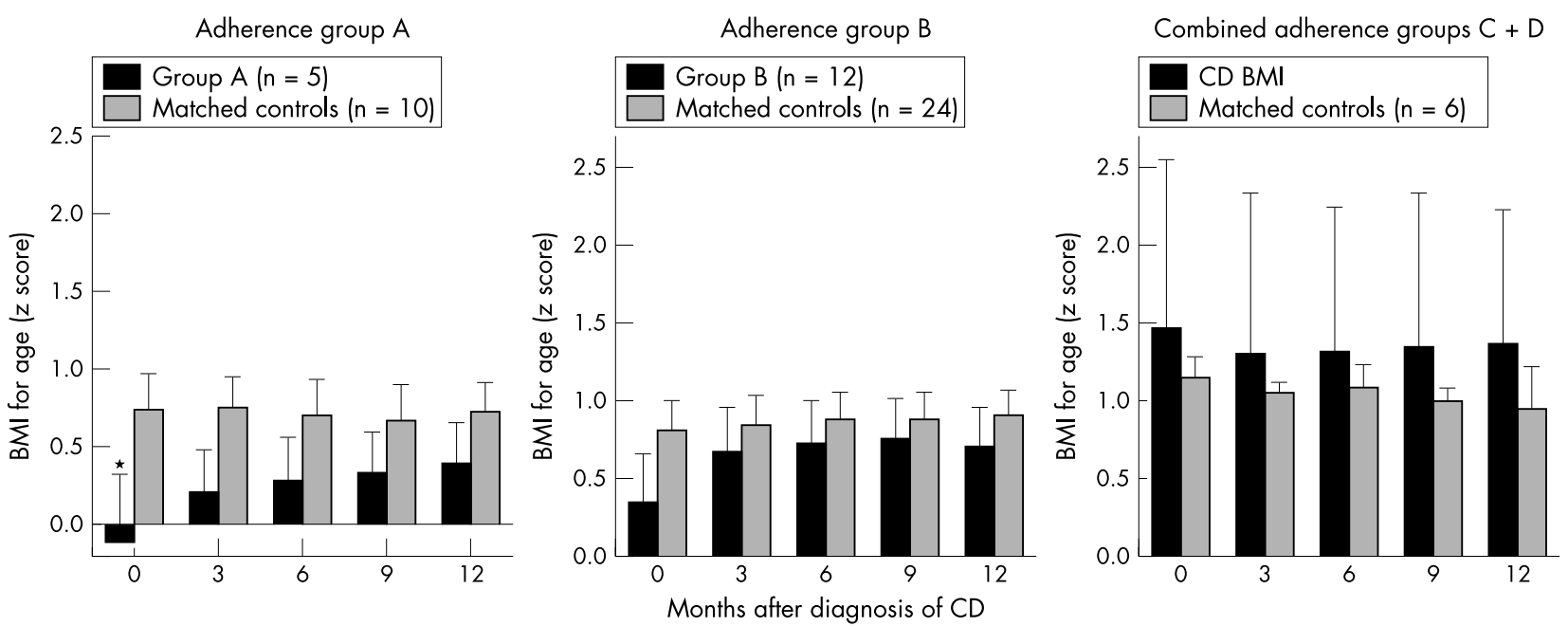

Figure 5 BMI-for-age after diagnosis of coeliac disease by dietary adherence. " $A$ " represented excellent adherence, " $B$ " good adherence, and "CD" was assessed as poor to fair. ${ }^{*} p<0.05$ compared to controls. 
previous studies in diabetic coeliacs of the effects of a glutenfree diet on growth and diabetic control have made no apparent attempt to determine dietary adherence or awareness. ${ }^{10}$ Although one of our chief aims was to compare growth and diabetic control between groups with varying degrees of dietary adherence, the small numbers of children within each group, and in particular the "fair" or "poor" adherence groups prevented a statistically meaningful analysis of their growth. Serological measures are at present still inadequate as a means of accurately assessing ongoing gluten ingestion. ${ }^{19}{ }^{20} \mathrm{~A}$ difficulty in comparing adherence rates between studies in coeliac disease is the lack of any standardised tools for assessment of adherence to a glutenfree diet. We developed a structured questionnaire to evaluate awareness of gluten content in food, gluten intake, parental knowledge of CD, and membership of the Coeliac Association. These factors have previously been shown to be predictive in determining dietary adherence. ${ }^{14}$ The timing of our questionnaire varied widely, ranging from 3 months to 13 years after diagnosis of CD. Studies which include larger numbers of poorly adherent coeliacs will be required to confirm our hypothesis that individual dietary adherence influences growth and to validate the questionnaire. It will also be important in future studies to repeatedly re-evaluate dietary adherence.

In our study, although patients identified with $C D$ were slightly smaller than matched controls, this difference failed to reach statistical significance and only a few had easily recognisable prior growth impairment. As a group, their mean weight, height and BMI-for-age were above the reference population mean. Despite this, diagnosis and treatment of $C D$ enhanced growth. These findings differ from those of previous studies. Acerini and colleagues ${ }^{12}$ found no significant improvement in BMI or in z scores for height in seven children with type 1 diabetes 12-24 months after diagnosis of CD. The authors concluded that the therapeutic value of dietary therapy in "asymptomatic" CD was uncertain. Westman and colleagues ${ }^{13}$ found that growth parameters were unaffected by dietary compliance in 20 diabetic coeliacs. Our study involved a slightly larger group than the studies of either Acerini and colleagues ${ }^{12}$ or Amin and colleagues. ${ }^{10}$ We also obtained longitudinal information after diagnosis in distinction to the cross sectional data reported by Westman and colleagues. ${ }^{13}$ The small number of patients with poor to fair adherence limited our ability to confirm any correlation between adherence and growth in this group. Nevertheless, we believe that dietary awareness and adherence are critical to evaluating the response in growth after starting a gluten-free diet. Outcome studies that do not take these into account are of doubtful validity.

We found that a gluten-free diet resulted in a significant increase in insulin dosage, but had no effect on HbAlc. Studies of the impact of diagnosis of coeliac disease on diabetic control have not produced clear results. Frequent hypoglycaemic episodes and a progressive reduction in insulin requirement have been reported as being suggestive of coeliac disease in patients with type 1 diabetes..$^{21}$ However, this has not been confirmed in a more recent study. Savilahti and colleagues ${ }^{11}$ showed no significant difference in insulin dose, HbAlc, or 24 hour urinary glucose excretion after GFD in seven children with both CD and type 1 diabetes. Acerini and colleagues ${ }^{12}$ found no significant differences in insulin dose, HbAlc, or number of hypoglycaemic episodes two years after starting a GFD in seven children with both CD and type 1 diabetes. The same result has also been found by Kaukinen and colleagues ${ }^{22}$ who reported no significant difference in metabolic control of diabetes in 28 adult patients studied retrospectively for three years before and five years after the introduction of GFD, and in 22 patients studied prospectively for a one year period after diagnosis of CD. Amin and colleagues, ${ }^{10}$ however, found that a gluten-free diet resulted in a significant fall in HbAlc, with no change in insulin dose.

The reasons for these varying findings on diabetic control are likely to be related to the complex impact of identification of coeliac disease in the diabetic patient. Intestinal absorption is certainly improved as villous atrophy resolves. However, the nature of the diet also has an impact. There is an inverse correlation between the glycaemic index of carbohydrates in the diet of diabetics ${ }^{23}$ and glycaemic control. Gluten-free pasta and bread have a much higher glycaemic index than gluten containing equivalents. Poor dietary adherence to a gluten-free diet is likely to influence diabetic control. More subtle markers of improved control are often difficult to measure. These include continuous blood glucose, fluctuations in blood glucose levels, and frequency of changes in insulin dosage. Further prospective studies that included detailed analyses of mean daily excursions of blood glucose for 12 month periods before and after the diagnosis of CD would help but would be logistically difficult.

It is important to recognise that untreated CD has many other well recognised long term consequences. These include osteoporosis, ${ }^{24}$ and an increased mortality from lymphoma and other malignancies. ${ }^{25}$ These can be prevented by a strict gluten-free diet. ${ }^{26}{ }^{27}$ We have shown benefit in terms of growth that may correlate with dietary adherence to a glutenfree diet. The relatively small number of those with CD clearly indicates that our findings need to be considered with due caution. Further carefully designed longitudinal studies are clearly needed, especially of those who were asymptomatic at diagnosis. Importantly, justification for identification of CD in type 1 diabetes and appropriate gluten restriction should also consider the long term health issues to these children.

\section{Conclusion}

Identification and dietary treatment of coeliac disease in children with diabetes improved growth and influenced diabetic control.

\section{Authors' affiliations}

O I Saadah, M R Oliver, A G Catto-Smith, Royal Children's Hospital, Department of Gastroenterology and Clinical Nutrition, Melbourne, Australia

M Zacharin, Royal Children's Hospital, Department of Endocrinology and Diabetes, Melbourne, Australia

A O'Callaghan, Royal Children's Hospital, Department of Nutrition and Food Services, Melbourne, Australia

\section{REFERENCES}

1 Walker-Smith JA, Vines R, Grigor W. Coeliac disease and diabetes. Lancet 1969;2:650.

2 Barera G, Bianchi C, Calisti L, et al. Screening of diabetic children for coeliac disease with antigliadin antibodies and HLA typing. Arch Dis Child 1991;66:491-4.

3 Gadd S, Kamath KR, Silink M, et al. Co-existence of coeliac disease and insulin-dependent diabetes mellitus in children: screening sera using an ELISA test for gliadin antibody. Aust N Z J Med 1992;22:256-60.

4 Sigurs N, Johansson C, Elfstrand PO, et al. Prevalence of coeliac disease in diabetic children and adolescents in Sweden. Acta Paediatr 1993;82:748-51.

5 Pocecco M, Ventura A. Coeliac disease and insulin-dependent diabetes mellitus: a causal association? Acta Paediatr 1995;84:1432-3.

6 Saukkonen T, Savilahti E, Reijonen $\mathrm{H}$, et al. Coeliac disease: frequent occurrence after clinical onset of insulin-dependent diabetes mellitus. Childhood Diabetes in Finland Study Group. Diabet Med 1996;13:464-70.

7 DeVitis I, Ghirlanda G, Gasbarrini G. Prevalence of coeliac disease in type I diabetes: a multicentre study. Acta Paediatr 1996;412(suppl):56-7.

8 Cronin CC, Shanahan F. Insulin-dependent diabetes mellitus and coeliac disease. Lancet 1997;349:1096-7.

9 Pena AS, Garrote JA, Crusius JB. Advances in the immunogenetics of coeliac disease. Clues for understanding the pathogenesis and disease heterogeneity. Scand J Gastroenterol Suppl 1998;225:56-8.

10 Amin R, Murphy N, Edge J, et al. A longitudinal study of the effects of a gluten-free diet on glycemic control and weight gain in subjects with type 1 diabetes and celiac disease. Diabetes Care 2002;25:1117-22. 
11 Savilahti E, Simell O, Koskimies S, et al. Celiac disease in insulin-dependent diabetes mellitus. J Pediatr 1986;108:690-3.

12 Acerini CL, Ahmed ML, Ross KM, et al. Coeliac disease in children and adolescents with IDDM: clinical characteristics and response to gluten-free diet. Diabet Med 1998;15:38-44

13 Westman E, Ambler GR, Royle M, et al. Children with coeliac disease and insulin dependent diabetes mellitus - growth, diabetes control and dietary intake. J Pediatr Endocrinol Metab 1999;12:433-42.

14 Jackson PT, Glasgow JF, Thom R. Parents' understanding of coeliac disease and diet. Arch Dis Child 1985;60:672-4.

15 Mayer M, Greco L, Troncone R, et al. Compliance of adolescents with coeliac disease with a gluten free diet. Gut 1991;32:881-5.

16 Colaco J, Egan-Mitchell B, Stevens FM, et al. Compliance with gluten free diet in coeliac disease. Arch Dis Child 1987;62:706-8.

17 Kumar PJ, Walker-Smith J, Milla P, et al. The teenage coeliac: follow up study of 102 patients. Arch Dis Child 1988;63:916-20.

18 Ljungman G, Myrdal U. Compliance in teenagers with coeliac disease-a Swedish follow-up study. Acta Paediatr 1993;82:235-8.

19 Dickey W, Hughes DF, McMillan SA. Disappearance of endomysial antibodies in treated celiac disease does not indicate histological recovery. Am J Gastroenterol 2000;95:712-14.
20 Hoffenberg EJ, Bao F, Eisenbarth GS, et al. Transglutaminase antibodies in children with a genetic risk for celiac disease. J Pediatr 2000;137: 356-60.

21 lafusco D, Rea F, Prisco F. Hypoglycemia and reduction of the insulin requirement as a sign of celiac disease in children with IDDM. Diabetes Care 1998;21:1379-81.

22 Kaukinen K, Salmi J, Lahtela J, et al. No effect of gluten-free diet on the metabolic control of type 1 diabetes in patients with diabetes and celiac disease. Retrospective and controlled prospective survey. Diabetes Care 1999;22:1747-8

23 Buyken AE, Toeller M, Heitkamp G, et al. Carbohydrate sources and glycaemic control in type 1 diabetes mellitus. EURODIAB IDDM Complications Study Group. Diabet Med 2000;17:351-9.

24 Kemppainen $\mathrm{T}$, Kroger $\mathrm{H}$, Janatuinen $\mathrm{E}$, et al. Osteoporosis in adult patients with celiac disease. Bone 1999;24:249-55.

25 Holmes GK. Celiac disease and malignancy. J Pediatr Gastroenterol Nutr 1997:24:S20-3

26 Valdimarsson T, Lofman O, Toss $G$, et al. Reversal of osteopenia with diet in adult coeliac disease. Gut 1996;38:322-7.

27 Holmes GK, Prior P, Lane MR, et al. Malignancy in coeliac disease-effect of a gluten free diet. Gut 1989;30:333-8.

\section{IMAGES IN PAEDIATRICS}

\section{Oesophageal dislocation of a percutaneous endoscopic gastrostomy (PEG)}

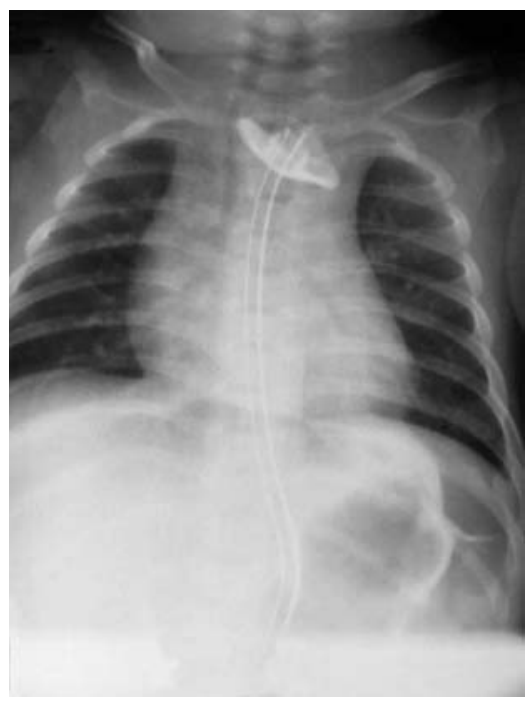

P Borusiak, P Gerner

Zentrum für Kinder- und Jugendmedizin, Helios Klinikum Wuppertal-Kooperierende Klinik der Universität Witten/Herdecke, Heusnerstr. 40, D-42283 Wuppertal, Germany; pborusiak@wuppertal.helios-kliniken.de 